\title{
The SPLIT Research Agenda 2013
}

\author{
Alonso EM, Ng VL, Anand R, Anderson CD, Ekong UD, Fredericks \\ EM, Furuya KN, Gupta NA, Lerret SM, Sundaram S, Tiao G, on \\ behalf of the Studies of Pediatric Liver Transplantation (SPLIT) \\ Research Group. The SPLIT Research Agenda 2013.
}

Abstract: This review focuses on active clinical research in pediatric liver transplantation with special emphasis on areas that could benefit from studies utilizing the SPLIT infrastructure and data repository. Ideas were solicited by members of the SPLIT Research Committee and sections were drafted by members of the committee with expertise in those given areas. This review is intended to highlight priorities for clinical research that could successfully be conducted through the SPLIT collaborative and would have significant impact in pediatric liver transplantation.

\author{
Estella M. Alonso ${ }^{1}$, Vicky L. $\mathbf{N g}^{2}$, \\ Ravinder Anand ${ }^{3}$, Christopher D. \\ Anderson ${ }^{4}$, Udeme D. Ekong ${ }^{1}$, Emily M. \\ Fredericks ${ }^{5}$, Katryn N. Furuya ${ }^{6}$, Nitika A. \\ Gupta ${ }^{7}$, Stacee M. Lerret ${ }^{8}$, Shikha \\ Sundaram ${ }^{9}$, Greg Tiao ${ }^{10}$ and on behalf of \\ the Studies of Pediatric Liver \\ Transplantation (SPLIT) Research Group \\ ${ }^{1}$ Department of Pediatrics, Ann \& Robert H. Lurie \\ Children's Hospital of Chicago, Chicago, IL, USA, \\ ${ }^{2}$ SickKids Transplant Center, The Hospital for Sick \\ Children and University of Toronto, Toronto, ON, \\ Canada, ${ }^{3}$ The EMMES Corporation, Rockville, MD, \\ USA, ${ }^{4}$ Division of Transplant and Hepatobiliary \\ Surgery, University of Mississippi Medical Center, \\ Jackson, MS, USA, ${ }^{5}$ Division of Child Behavioral \\ Health, Department of Pediatrics and Communicable \\ Diseases, University of Michigan, Ann Arbor, MI, \\ USA, ${ }^{6}$ Department of Pediatrics, Thomas Jefferson \\ University, Philadelphia, PA, USA, ${ }^{7}$ Department of \\ Pediatrics, Emory University School of Medicine, \\ Atlanta, GA, USA, ${ }^{8}$ Department of Pediatrics, \\ Medical College of Wisconsin, Milwaukee, WI, USA, \\ ${ }^{9}$ Pediatric Liver Center and Section of Pediatric \\ Gastroenterology, Hepatology and Nutrition, \\ Department of Pediatrics, University of Colorado \\ Denver School of Medicine, Children's Hospital \\ Colorado, Denver, CO, USA, ${ }^{10}$ Departments of \\ Pediatric and Thoracic Surgery, Cincinnati Children's \\ Hospital and Medical Center, Cincinnati, OH, USA \\ Key words: outcome - pediatric liver \\ transplantation - indications - long-term results - \\ health outcomes \\ Estella M. Alonso, Department of Pediatric \\ Gastroenterology, Ann \& Robert H. Lurie Children's \\ Hospital of Chicago, 225 E Chicago, Box 65, Chicago, \\ IL 60611, USA \\ Tel.: $312-227-4606$ \\ Fax: 312-227-9645 \\ E-mail: ealonso@luriechildrens.org \\ Accepted for publication 11 April 2013
}

SPLIT is a community of pediatric hepatologists, transplant surgeons, research coordinators, nurse coordinators, and other health professionals across the United States and Canada working together to advance knowledge in pediatric liver transplantation. SPLIT was founded in 1995 and

Abbreviations: COG, Children's Oncology Group; DRI, donor risk index; GI, gastrointestinal; GPOH, German Society for Pediatric Hematology/Oncology; HAT, hepatic artery thrombosis; HB, hepatoblastoma; HCC, hepatocellular carcinoma; IS, immunosuppression; ITN, immune tolerance network; iWITH, Immunosuppression Withdrawal for Stable Pediatric Liver Transplant Recipients; LT, liver transplant; MELD, model for end-stage liver disease; NIDDK, National Institute of Diabetes and Digestive and Kidney Diseases; PLUTO, Pediatric Liver Unresectable Tumor Observatory; PRETEXT, Pretreatment extent of disease; PTLD, post-transplant lymphoproliferative disease; SIOPEL, Liver Tumor Strategy Group of the International Society of Pediatric Oncology; SPLIT, The Studies of Pediatric Liver Transplantation; TLCT, transitional liver cell tumor. 
has evolved from a research registry into a multifaceted organization focused on improving outcomes for children receiving liver transplantation. The original support for registry development and data collection was provided by industry partners with interest in the area of transplantation. The focus at that time was on collecting descriptive data regarding candidate characteristics, surgical approaches, and key outcomes including patient and graft survival, rejection and vascular complications. The data were designed to be more comprehensive than what was collected for the United Network for Organ Sharing and the data collection extended into long-term follow-up.

As registry participation and the patient cohort grew, leaders of the SPLIT Research group developed a set of aims and hypotheses for ongoing research that were informed by early registry findings. These aims became the core of a proposal that was funded by the NIDDK as a five-yr consortium grant, during which time 1334 transplant recipients were newly enrolled. Federal funding of the consortia extended for a six-yr period, and analyses of registry data resulted in 22 peer reviewed publications. At the peak of enrollment, the registry included 45 centers in the United States and Canada, and data collection was captured for approximately $71 \%$ of the pediatric liver transplants that occurred each year in North America. Data collected during this time period form an important repository that will inform decisions in pediatric transplantation for many years to come.

In 2009, the research group underwent a transformative period realigning their priorities and funding mechanisms to meet current challenges in health outcomes research. During this transformation, the group's goals were extended beyond that of clinical research to embrace the missions of clinician education and patient advocacy. The group now continues to collect abbreviated registry data on newly transplanted patients and long-term follow-up information for patients in the existing cohort at currently participating SPLIT centers. Current research questions focus on quality initiatives, and data collection is tailored on an ongoing basis to support current research questions and priorities. The existing registry allows participating investigators to easily identify potential candidates for intervention trials and thus provides a valuable infrastructure for new grant development. Each participating center contributes funding to support the data coordinating center and underwrites the efforts of research coordinators and nurses to submit data.
The purpose of this document is to define research priorities for the SPLIT group as the effort moves forward. This review focuses on active clinical research in pediatric liver transplantation with special emphasis on areas that could benefit from studies utilizing the SPLIT infrastructure and data repository. Ideas were solicited by members of the SPLIT Research Committee, and sections were drafted by members of the committee with expertise in those given areas. This review is intended to highlight priorities for clinical research that could successfully be conducted through the SPLIT collaborative and would have significant impact in pediatric liver transplantation.

\section{Strategies to improve early graft function}

\section{Development of a pediatric-specific DR}

The development of the concept of a DRI in liver transplantation represented an advance in the field as it clearly defined donor characteristics that were associated with future allograft survival (1). Equally as important, it formed a foundation for other studies that have significantly advanced the field. While the DRI is an important variable in liver transplantation, it does have clear limitations. For example, despite the increasing epidemic of non-alcoholic fatty liver disease, there is currently no clearly defined mechanism in which to incorporate hepatic steatosis into the DRI. Further, compared with candidate disease severity, donor quality is only a relative consideration. A donor with an unacceptably high DRI for a patient with a relatively low MELD score may be a perfectly appropriate option and offer a significant survival advantage for a patient with a higher MELD score (2).

Many of the variables included in the DRI have been discussed in the pediatric liver transplant literature. For example, donor age and the use of technical variant allografts have been shown by many authors to carry an increased relative risk of graft failure in children (3-5). However, these risks are only a relative consideration compared with recipient disease severity. While the global DRI, in general, can be adopted for the pediatric population, the fidelity of prediction likely decreases. In addition, given the many unique pediatric diseases treated with liver transplantation, the development of a pediatric-specific DRI is an identifiable need. The SPLIT Research group is ideally poised to support such a research project. The ability to define multiple donor variables matched to pediatric-specific problems such as recipient size, diagnoses, and disease severity 
can more clearly define the combination of donor and recipient outcome predictors.

One advantage of a pediatric-specific DRI is the ability to better predict early allograft function. There are many reasons to devote research effort toward improvements in this area. First and foremost is the improvement of graft function and survival in pediatric recipients. In this area, a better understanding of the relationship between immediate allograft function and longterm graft and patient survival would be useful. Second, it is clear that allografts with delayed functional recovery lead to increased resource utilization and increased costs. The ability to predict early functional recovery of an allograft is an area in which the SPLIT study group should strive to involve basic and translational science into DRI predictions. Much like the development of a reliable steatosis predictor could improve the adult DRI, incorporation of a measurable physiologic donor metric or a serum biomarker of liver injury will significantly increase the fidelity of any pediatric-specific DRI.

\section{Potential research projects}

1 Registry-driven study to determine donor risk factors that determine early allograft function in pediatric liver transplant recipients with emphasis on factors that are important to the function of technical variant grafts. Such an analysis would evaluate the relative impact of such factors across different recipient disease states.

Technical complications following pediatric liver transplantation

Much is written about complications following pediatric liver transplantation. The reported rates vary greatly depending on the time of follow-up reported, the type of allograft used, and the age/size of the recipient. A recent report from the SPLIT study group offers the sobering statistic that $71.4 \%$ of pediatric recipients of a whole organ liver transplant will have a complication within two yr of transplant. This percentage grows as high as $86.9 \%$ for recipients of technical variant allografts (4). While these statistics incorporate many measured complications, biliary, vascular, and GI complications are major contributors to post-transplant morbidity in pediatric recipients. There are many studies in the current literature describing single-center approaches to many of these complications; however, there are little prospective data evaluating various strategies designed to prevent or treat these complications.
A review of the literature regarding vascular thrombosis following pediatric liver transplantation reveals myriad manuscripts discussing the use of operating microscope vs. loupe magnification, the use of differing techniques of vascular reconstruction, routine vs. selective use of Doppler ultrasound, and the use of various anticoagulation protocols. Given that these are usually single-center studies with varying baseline rates of vascular thrombotic complications and differing patient demographics (age/size), it is difficult to determine a "best practice" strategy. The average rate of HAT at centers participating in the SPLIT study group is between $4 \%$ and $9 \%$ (4), and it is difficult to imagine being able to demonstrate a significant reduction in rates of HAT without involving multiple centers in a cooperative effort. For this and other reasons, the SPLIT study group is uniquely poised to be the leader in this effort.

Similar analogies can be made for biliary complications which have a much higher occurrence, especially in recipients of technical variant allografts $(4,6)$. Given that the rates of these types of technical complications have changed little in the last $10 \mathrm{yr}$, coordinated, multicenter studies addressing these problems in pediatric liver transplantation will be needed to advance the field. These studies will ideally be a combination of clinical, basic, and translational science.

\section{Potential research projects}

1 Randomized trial of anticoagulation protocols measuring the combined end points of portal vein or hepatic arterial thrombosis and re-exploration for intraperitoneal bleeding within the first $72 \mathrm{~h}$ following reperfusion. Designing such a study would require a careful survey of standard practices across SPLIT centers with the expectation that some form of therapy is standard. Thus, the study would randomize patients to receive one of two commonly used protocols to compare the risks and benefits.

2 Longitudinal analysis of outcomes following percutaneous treatment of biliary strictures. Such a study could be conducted as an observational analysis with the primary end point being clinical evidence of stricture recurrence in 36 months following initial therapy. The analysis would examine method and duration of stent placement, currently not standardized, as predictors of the outcome. Risks would be adjusted for primary diagnosis, type of graft, history of hepatic arterial complication, and area of stricture (anastomosis vs. intrahepatic). 


\section{Refining indications for transplant in special populations}

Hepatic malignancy in children

The two most common malignant liver tumors in children are $\mathrm{HB}$ and $\mathrm{HCC}$. In patients diagnosed with $\mathrm{HB}$, the standard treatment algorithm employs neoadjuvant chemotherapy followed by resection; however, in a subgroup of patients, tumor burden precludes conventional resection. In this subgroup, liver transplantation is used to achieve local control. In children diagnosed with $\mathrm{HCC}$, the role of chemotherapy remains controversial; complete local control by conventional resection or liver transplantation is the only route to achieve long-term cure. Ongoing efforts by the COG and the SIOPEL to identify the appropriate indications for transplantation could be enhanced with additional research efforts conducted by the SPLIT consortium.

\section{Hepatoblastoma}

HB accounts for about $80 \%$ of the malignant liver tumors in children. The incidence has increased from 0.6 to 0.8 per million to $1.2-1.5$ per million over the past two decades $(7,8)$. PRETEXT, a staging system devised by the SIOPEL in the 1990s (9) and revised for SIOPEL 3 in 2007 (10), is based on cross-sectional imaging of the extent of tumor, and assignment to one of the four PRETEXT groups (PRETEXT I, II, III, or IV) is determined by the number of contiguous uninvolved sections of the liver. PRETEXT is further annotated with a V, P, E, M, or C depending upon extension of tumor beyond the hepatic parenchyma of the major sections. In the current trials, surgical resection guidelines use PRETEXT to define which tumors should be resected at diagnosis, receive neoadjuvant chemotherapy, and which should be referred for possible liver transplantation. Complete eradication of tumor is a prerequisite for cure which makes an aggressive surgical approach necessary and highlights one of the advantages of primary transplantation. Contrary to earlier trials where decisions about surgical resection were made by individual surgeons, the surgical guidelines of the current COG trials use PRETEXT to define the timing and extent of surgical resection, the use of neoadjuvant chemotherapy, and indications for liver transplantation. Resection at diagnosis is recommended only when a segmentectomy or non-extended lobectomy will predictably yield a complete resection, and neoadjuvant therapy is given prior to resection of PRETEXT III tumors. Alternatively, in the European SIOPEL and GPOH study groups, neoadjuvant chemotherapy is given to all patients with the rare patient going directly to transplant depending upon the recommendation of the transplant center.

After the pioneering work of Reyes, Superina, and Al Qabandi in the late 1990s (11-13), transplantation has become a critical component of the treatment algorithm of $\mathrm{HB}$. Long-term survival ranging from $55 \%$ to $100 \%$ has been reported over the past decade in over a dozen single centers. Cases of "unresectable" HB due to involvement of the entire liver, extensive multifocality, or major hepatic venous or portal venous involvement comprise $10-20 \%$ of all HB treated in multicenter trials. The best results for highrisk HB reported to date were in SIOPEL 3 (14), and the improvement in outcome seen in this study appears to be at least partly due to an increase in the use of liver transplant. Multiple series have shown superior outcome with primary transplant (about $80 \%$ overall survival) compared with rescue transplant (about 30-40\% overall) (15-20). The basis for this is undoubtedly multifactorial, but two important concerns are the likelihood of chemotherapy resistance in relapse tumors $(21,22)$, and the debilitated state of the patients when transplanted in the face of end-stage disease.

SIOPEL, together with support from COG, GPOH, SPLIT, and individual pediatric liver transplant centers all over the world, has established a worldwide electronic registry for liver transplant for childhood tumors (HB, HCC, infantile hemangioma, and others) titled the PLUTO (23). This collaboration will allow assessment of outcomes across an international cohort and could provide infrastructure for valuable treatment trials that explore the benefits of LT in children with extensive HB.

\section{Hepatocellular carcinoma}

HCC occurs predominantly in the setting of underlying liver disease and cirrhosis. However, de novo tumors without underlying cirrhosis are more common in children than adults (24). More than two-thirds of pediatric HCC occur in children older than $10 \mathrm{yr}$ of age, but only $0.5-1 \%$ of all HCC manifest before $20 \mathrm{yr}$ of age. Very few HCCs are diagnosed in children less than five yr old, and in this young age group, transitionaltype tumors are seen. About $20-35 \%$ of children with HCC have underlying chronic liver disease. It is still disputed whether "adult-type" HCC in children is the same or a different disease. Zimmermann and others have suggested that HCC forms a tumor family, consisting of adult-type $\mathrm{HCC}$ and its variants, fibrolamellar HCC, and a 
novel entity occurring in young children and adolescents, TLCT $(25,26)$.

No staging or grading system has been found that accurately predicts prognosis in pediatric HCC. In the pediatric multicenter trials, HCC has been usually treated using the same protocols, but analyzed separately. PRETEXT has been used because of its utility in $\mathrm{HB}$ and the crossover between these two tumors in the intermediate age group. HCC is relatively chemoresistant and therefore carries a poor prognosis with a dismal cure rate $(27,28)$. Complete surgical resection or transplantation of tumor localized to the liver is often the only hope. Unfortunately, $\mathrm{HCC}$ is most often advanced at diagnosis and cure is rarely possible in the setting of metastatic disease. The main prognostic factor for childhood HCC is resectability.

The role of liver transplantation in pediatric $\mathrm{HCC}$ is in greater evolution than in pediatric HB. Liver transplant is contraindicated in the presence of any extrahepatic tumor, even in the occasional patient who regresses with chemotherapy. Some argue an exception might be made in the intermediate case of children with TLCT. Outcome for transplant in adult HCC has improved over the years due to the recognition that strict selection criteria are important in preventing post-transplant tumor relapse. The Milan criteria, introduced by Mazzaferro in 1996 for adults with advanced cirrhotic liver disease, were developed for a patient population, who develop tumor nodules (usually multiple) as part of their cirrhotic (often alcoholic) liver disease. The Milan criteria restrict transplant for HCC in adults to: (i) single tumor $<5 \mathrm{~cm}$; (ii) not more than three nodules; (iii) no angioinvasion; (iv) no extrahepatic involvement (29). The problem with applying the Milan criteria to children is that 50 $70 \%$ of children present with large de novo tumors in an otherwise healthy (non-cirrhotic) liver. These de novo tumors in non-cirrhotic livers in children are felt to exist on a continuum with transitional cell tumors and $\mathrm{HB}$, and seem to have a different biology (30), at the very least they have a very different epidemiology. Two recent series of pediatric liver transplant questioned the relevance of Milan criteria to pediatric HCC. In a series from Stanford, 10 children were transplanted for HCC and neither the number of tumors, nor the size of tumor, nor the presence of gross vascular invasion was correlated with the risk of post-transplant tumor relapse (31). Of the four Milan criteria evaluated in children in a transplant series from Poland: three children did not fulfill four criteria; three children did not fulfill two criteria; and two children did not fulfill one criterion (32). Thus, it is unclear whether children with HCC should be limited to the same treatment options offered to adults (33).

\section{Potential research projects.}

In the current protocols conducted by COG and SIOPEL, liver transplantation is the end point of the algorithm. The goal of PLUTO is to begin to describe the outcomes following transplantation; however, as a registry, it faces inherent challenges. In that respect, there are multiple opportunities for the SPLIT consortium to contribute.

These include:

1 Registration of all patients transplanted for hepatic malignancy at SPLIT centers into the PLUTO registry to coordinate longitudinal observational studies.

2 Prospective randomized trials evaluating the role of immunosuppression in the context of chemotherapy.

3 Outcomes comparisons between conventional resection vs. liver transplantation to define the benefits of transplantation.

\section{Issues in maintenance of long-term graft function}

Tolerance in pediatric liver transplantation

Pediatric liver transplant recipients are at risk to develop multiple complications related to longterm exposure to IS. These complications include immune and non-immune related illnesses, many of which could be life threatening (opportunistic infection and PTLD) or lead to chronic disease that can limit the patient's life span (renal insufficiency and diabetes).

Single-center experiences in which patients are withdrawn from immunosuppression due to medical complications such as PTLD or renal insufficiency suggest that approximately $20 \%$ of LT recipients are functionally tolerant. Experience at the University of Pittsburgh (both published and unpublished) suggests a higher success rate for pediatric recipients with an observed operational tolerance rate in $22(34 \%)$ of 64 recipients (34). The Kyoto University transplant program has similarly reported that $15 \%$ of their entire, unselected cohort of 581 pediatric living donor liver recipients has been withdrawn from IS with $<1 \%$ of patients developing obvious chronic rejection $(35,36)$. This experience informed a recent phase I trial conducted through the ITN in which 20 pediatric LT recipients of parental living donor transplant were withdrawn from immunosuppression over a 12month period. In this trial, 12 of the 20 patients remain off IS at 20-41 months with stable graft 
function and no histological evidence of chronic rejection and no episodes of graft loss (37). As a safety study, this trial did not include a control arm and was not powered to examine significant changes in histology over time. Although acute cellular rejection was not diagnosed in the tolerant group, many had liver dysfunction and mild inflammatory changes on liver biopsy. Inclusion of a control arm and examination of a larger sample size in future studies are essential to determine the durability of tolerance in this group and better define inflammatory and afibrotic changes in the grafts over time.

Ongoing tolerance trials are dependent upon the principle that acute rejection episodes can be easily reversed without permanent injury to the graft. Current IS withdrawal or minimization study designs employ a "trial and error" approach, gradually eliminating IS until the patient develops signs of acute rejection. Widespread clinical application of IS withdrawal will depend upon the ability to reliably predict which patients will develop operational tolerance, thus minimizing the risk of acute rejection (38). Clinical (interval from transplant) and histological features (lack of graft inflammatory changes) suggest a phenotype of patient most likely to safely withdraw from IS, but there are no accepted methods to monitor the patient's functional immune status or level of reactivity to the liver allograft (39). Identification of a reproducible fingerprint of operational tolerance is a prerequisite for broad application of IS drug withdrawal in liver transplant recipients. Even though many immune monitoring techniques have been developed with potential to detect active mechanisms of immune tolerance or preclinical signs of allograft rejection, no assay has been prospectively shown to serve as a robust biomarker of operational tolerance.

\section{Potential research projects}

1 Randomized controlled trial of IS withdrawal in pediatric patients. Such a study would include collection of biospecimens for immune monitoring and serial liver histology (see below). Analysis of clinical predictors of tolerance would be included as well. Similar to this, iWITH is a recently launched multicenter, open label, longitudinal, phase II clinical trial to determine the safety and efficacy of immunosuppression withdrawal with concomitant portfolio of translational studies to develop and validate a fingerprint which predicts operational tolerance. This study currently involves 11 pediatric liver transplant centers/clinical sites in the United States and Canada. The primary goal of this study is to test the hypothesis that a defined subset of stable pediatric liver transplant recipients can safely and durably withdraw from immunosuppression (37).

2 Large-scale (sample size 150-200 patients) study to examine histological response to immunosuppression withdrawal which includes a control arm and allows histological comparisons over time.

3 Development of immune monitoring techniques to identify tolerant patients.

Long-term graft injury after pediatric liver transplantation

Histological abnormalities are commonly present in late post-transplant biopsies, including protocol biopsies from patients who appear to be well with good graft function. Some of the abnormalities seen include rejection (which may have a different appearance from rejection seen in the early post-transplant period), de novo disease, idiopathic post-transplant allograft hepatitis, allograft fibrosis, nodular regenerative hyperplasia, vascular anomalies, and recurrent disease. The prevalence and spectrum of changes reported in these long-term biopsies varies between different transplant centers. This difference may be reflective of center-specific immunosuppression practice, as well as differences in the terminology used to describe changes of uncertain etiology in late biopsies.

The use of protocol biopsies has changed in recent years. While most centers use protocol biopsies to assess disease progression in chronic hepatitis $\mathrm{C}$ for instance, the majority have discontinued this practice in other transplant recipients. However, numerous studies have shown that histological abnormalities are not only frequently seen in protocol biopsies from recipients with normal liver enzymes (40, 41), but some of these abnormalities are potentially significant (40, 42-44).

\section{Potential research projects}

1 Longitudinal analysis of protocol biopsy findings in patients on minimal immunosuppression, i.e., once daily calcineurin inhibitors or rapamycin. This should include histological analysis of bile duct atypia/atrophy/focal loss or fibrosis.

2 Longitudinal analysis of the evolution of patients with allograft hepatitis, especially those classified as de novo alloimmune hepatitis.

3 The development of optimal algorithms for the use of liver biopsy in the assessment of the long-term liver allograft. 


\section{Improving health for the long-term survivor}

Long-term medical complications after pediatric liver transplantation

Long-term survival after pediatric liver transplantation is the rule rather than the exception. Patients and families and healthcare providers face major challenges particularly related to the life-long immunosuppression and follow up currently necessary post-transplant (45). These challenges pertain to both the numerous immuneand non-immune-mediated complications, risks of both over- and under-immunosuppression, as well as the "uncharted territory" of current practice of life-long immunosuppression required by our patients. Indeed, less than one-third $(32 \%)$ of patients achieved an "ideal" profile of a first allograft stable on immunosuppression monotherapy, normal growth, and absence of common immunosuppression-induced sequelae (46). Multiple published, in press, and in preparation SPLIT manuscripts provide most commonly a cross-sectional study analyses of some of these long-term medical complications (47-52).

While liver transplant programs still need to pay critical attention to short-term survival, the need to develop strategies which will further increase the proportion of "perfect patients" and decrease late allograft dysfunction (chronic hepatitis, fibrosis, and biliary "misery") is paramount. These efforts and other opportunities will ideally require the whole arsenal of clinical, basic, and translational science. Directed toward the prevention of complications, mitigation of early processes, and rescue of existing complications, this research calls for collaboration, energy, and strategies among individual SPLIT centers.

\section{Potential research projects}

1 Longitudinal assessment of outcomes for pediatric liver transplant recipients which couples clinical data acquisition with biological specimen collection. Such endeavors would require consistent and uniform approaches to data and specimen collection to enable the desired goal of well-characterized long-term patient cohorts, possibly extending such tracking into early adulthood, analogous to what has been done for cancer survivors.

2 Longitudinal observational studies of the relationship between cardiovascular risk/outcomes and immunosuppression medications. Potential considerations include: (i) linking risk factors for cardiovascular disease to abnormalities in intermediate end points such as arterial stiffness and carotid intima media thickness which may permit focused interventions in high-risk patients before development of clinical disease; (ii) earliest evaluation and potential treatment (whether directed lifestyle modification or pharmacological therapies) may be key to ensuring best outcomes; and (iii) metabolic syndrome.

3 Development of models to predict post-transplant renal function that include factors related to phenotype such as underlying renal disease and genetic polymorphisms that impact calcineurin inhibitor pharmacodynamics.

4 Risk assessment of post-transplant malignancies which might help define screening guidelines for malignancies in pediatric LT recipients. Recent SPLIT cross-sectional analyses might serve as background and pilot data for specific initiatives.

5 Assessment of the impact of chronic anemia on health status and fatigue.

6 Assessment and evaluation of health-related quality of life in selected patient cohorts including possibly adult survivors of pediatric liver transplantation.

Transition and adolescent issues after pediatric liver transplantation

The adolescent developmental period is characterized by change in cognitions, emotional attachments, physical development, independence, education/vocation, and self-identity. This stage of development is a critical period for the establishment of both lifelong positive and risky health-related behaviors. With respect to chronic illness, disease management, including medication adherence, is often at odds with typical adolescent development $(53,54)$. The developmental characteristics associated with adolescence, including developing autonomy from family, assimilating with peers and separating from parents, poorly developed abstract thinking and understanding long-term consequences of present actions, are often difficult to balance with the behaviors required for optimal medication adherence (55).

The prevalence of non-adherence among pediatric transplant recipients ranges from 5 to $80 \%$, with adolescents having the highest rates of nonadherence (56-58). These rates of non-adherence are striking given the potential for serious consequences including graft rejection, graft loss, posttransplant mortality, poor health-related quality of life, and increased healthcare costs (59-63). Medication non-adherence has also been implicated in poor health outcomes following the transfer from pediatric to adult-centered care 
(64). Given that adolescents are at high risk of non-adherence and associated late graft dysfunction, interventions aimed at promoting medication adherence in this population are urgently needed.

Yet, before interventions targeting adherence can be implemented, it is necessary to incorporate adherence assessment into standard clinical care (65). Unfortunately, to date, studies of medication adherence in pediatric transplantation are limited by a lack of an accepted "gold standard" method for assessing adherence. Recent studies have attempted to measure adherence objectively using the degree of fluctuation (i.e., standard deviation, s.d.) of medication blood levels of tacrolimus in pediatric liver transplant recipients (59, 66-68). Higher s.d.s were predictive of clinical outcomes, such as biopsy-proven rejection and hospitalizations.

"Transition" is an active process that addresses the medical, psychosocial, and educational/vocational needs of adolescents as they prepare to move from child- to adult-centered health care (69), while "transfer" refers to the change in the location where care is provided $(70,71)$. The transition process is twofold as it includes the transition of responsibility for healthcare tasks from the parent to the patient, as well as the preparation to transfer to adult-centered care. Beginning in late childhood/early adolescence, the management of a chronic illness begins to shift from the primary responsibility of the parent to self-management by the adolescent (72-74). By shifting responsibility for health-related tasks in a developmentally appropriate manner, the adolescent gains the knowledge, skills, and experiences necessary to master the independence required to be successful in the adult healthcare system.

Self-management skills are integral to the achievement of independence necessary for successful healthcare transitions $(73,75)$. Researchers and clinicians agree that adolescents and young adults should not transfer from pediatric to adult health services unless they have the skills necessary for functioning effectively in the adult healthcare system, including adhering to medication regimens $(75,76)$. The Pediatric Committee of the American Society of Transplantation has recommended that prior to transferring to adultcentered care, the pediatric transplant recipient should demonstrate the ability to independently manage their health (77). In addition, the pediatric patient should adhere to their immunosuppressant medications to avoid increased risk of graft loss and rejection following the transfer to adult-centered care $(63,64)$.

\section{Potential research projects}

\section{Adolescent issues.}

1 Assessment of the impact of executive functioning on medication adherence and transition readiness among adolescents.

\section{Transition planning.}

1 There is a critical need for the development and validation of objective assessment tools to empirically evaluate the pediatric patient's readiness to move from a pediatric to adultfocused transplant health care. Transition readiness assessment tools should evaluate regimen knowledge, allocation of responsibility for healthcare tasks, self-management skills, and adherence.

2 Assessment of the impact of healthcare transition on measures of patient satisfaction, medical stability, quality of life, psychosocial functioning, educational/vocational outcomes, and healthcare utilization rates.

3 The timing of transfer from pediatric to adult-focused care should be individualized and based on the acquisition and mastery of self-management skills. Thus, research is needed to develop benchmarks to guide in determining the individualization of transfer to adult-centered care. The development of transition planning curricula and guidelines should include collaboration with the adolescent/young adult recipients and parents to determine how to best provide them with information related to the transition process.

4 Research is needed to define and identify predictors of successful transition to inform the development of programs which target modifiable factors. Research is needed to identify a standard transition practice for transplant providers to follow to enhance communication and collaboration between pediatric and adult caregivers during the transitional period.

5 Research investigating the role of health literacy and patient education in the transition process is also warranted.

\section{Adherence promotion and self-management inter- ventions.}

1 Future studies should continue to focus on developing a standardized method for routinely assessing medication adherence in pediatric liver transplant recipients. 


\section{Alonso et al.}

2 The development of empirically based interventions to promote self-management skills is critical. Interventions should also target parental monitoring and the transition of responsibility of health-related tasks from the parent to the adolescent/young adult. Thus, interventions should focus on the role of parental monitoring and supervision of medication-related tasks as adolescents begin to demonstrate mastery of health management tasks.

3 Healthcare providers are responsible for the delivery of health-related information, fostering motivation, assisting their patients with the behavioral skills necessary for adherence, and collaborating on plans for chronic illness management. Future research should examine the impact of physician communication and motivational strategies on medication adherence in adolescents.

4 Interventions delivered using newer technologies, such as cell phone text messages and the internet, may be promising for promoting medication adherence in adolescents. Further investigation of the sustainability and effectiveness of these innovative eHealth interventions is needed.

\section{Summary}

Great strides have been made in improving health outcomes in recipients of pediatric liver transplantation in the past $15 \mathrm{yr}$. The SPLIT Research group has been an invaluable spring board and infrastructure to support analyses that have informed and transformed care of this complex patient group. With the shifting sands of clinical research funding, the SPLIT group has reorganized to meet current challenges and continue in their mission to inspire and support innovative studies and initiatives to improve patient care.

\section{SPLIT Research Group}

Ann \& Robert H. Lurie Children's Hospital of Chicago, Chicago (Estella Alonso, MD).

University of Mississippi Medical Center, Jackson (Christopher Anderson, MD).

Mt. Sinai Medical Center, New York (Ronen Arnon, MD, MHA).

University of Chicago, Chicago (Ruba Azzam, MD).

Alfred I duPont Hospital for Children, Wilmington (Stephen Dunn, MD).

Ann \& Robert H. Lurie Children's Hospital of Chicago (Udeme Ekong, MD, MPH).

Boston Children's Hospital, Boston (Scott Elisofon, MD).

Children's Mercy Hospital and Clinics, Kansas City (Vicki Fioravanti, RN, CCTC).
University of Michigan, Ann Arbor (Emily Fredericks, $\mathrm{PhD})$.

Alfred I duPont Hospital for Children, Wilmington (Katryn Furuya, MD, FRCPC).

Emory-Children's Center, Atlanta (Nitika Arora Gupta, MD, DHC, DNB, MRCPCH).

The Hospital for Sick Children, Toronto (Binita Kamath, MBBChir, MRCP, MTR).

Children's Hospital of Wisconsin, Milwaukee (Stacee Lerret, $\mathrm{PhD}$ ).

University of Michigan, Ann Arbor (John Magee, MD).

Carolinas Medical Center, Charlotte (Jerome Menendez, MSN, FNP-C).

University of Chicago, Chicago (Michael Millis, MD).

The Hospital for Sick Children, Toronto (Vicky $\mathrm{Ng}$, MD).

Children's Hospital Colorado, Denver (Shikha Sundaram, MD, MSCI).

Cincinnati Children's Hospital and Medical Center, Department of Pediatric Surgery (Greg Tiao, MD).

Washington University School of Medicine, St. Louis (Yumirle Turmelle, MD).

Medical College of Wisconsin, Milwaukee (Bernadette Vitola, MD, MPH).

\section{References}

1. Feng S, Goodrich NP, BragG-Gresham JL, et al. Characteristics associated with liver graft failure: The concept of a donor risk index. Am J Transplant 2006: 6: 783-790.

2. Schaubel De, Sima CS, Goodrich NP, Feng S, Merion RM. The survival benefit of deceased donor liver transplantation as a function of candidate disease severity and donor quality. Am J Transplant 2008: 8: 419-425.

3. Anderson CD, Turmelle YP, Lowell JA, et al. The effect of recipient-specific surgical issues on outcome of liver transplantation in biliary atresia. Am J Transplant 2008: 8: 11971204.

4. Diamond IR, Fecteau A, Millis JM, et al. Impact of graft type on outcome in pediatric liver transplantation: A report From Studies of Pediatric Liver Transplantation (SPLIT). Ann Surg 2007: 246: 301-310.

5. Cacciarelli TV, Dvorchik I, Mazariegos GV, et al. An analysis of pretransplantation variables associated with long-term allograft outcome in pediatric liver transplant recipients receiving primary tacrolimus (FK506) therapy. Transplantation 1999: 68: 650-655.

6. Anderson CD, Turmelle YP, Darcy M, et al. Biliary strictures in pediatric liver transplant recipients - early diagnosis and treatment results in excellent graft outcomes. Pediatr Transplant 2010: 14: 358-363.

7. Mclaughlin CC, Baptiste MS, Schymura MJ, Nasca PC, ZDEB MS. Maternal and infant birth characteristics and hepatoblastoma. Am J Epidemiol 2006: 163: 818-828.

8. Bulterys M, Goodman M, Smith M, Jd B. Hepatic tumors. In: Ries LAG, Smith MA, Gurney JG, et al., eds. Cancer Incidence and Survival among Children and Adolescents: United States SEER Program 1975-1995. Bethesda, MD: National Cancer Institute, 1999.

9. Plaschkes J, Perilongo G, Shafford E, et al. SiOP trial report - overall preliminary results of SIOPEL-1 for the treatment of hepatoblastoma (HB) with pre-operative chemotherapy - continuous infusion cisplatin and doxorubicin (PLADO). 1994. 
10. Roebuck DJ, Aronson D, Clapuyt P, et al. 2005 PRETEXT: A revised staging system for primary malignant liver tumours of childhood developed by the SIOPEL group. Pediatr Radiol 2007: 37: 123-132; quiz 249-150.

11. Superina R, BiLik R. Results of liver transplantation in children with unresectable liver tumors. J Pediatr Surg 1996: 31: 835-839.

12. Reyes JD, Carr B, Dvorchik I, et al. Liver transplantation and chemotherapy for hepatoblastoma and hepatocellular cancer in childhood and adolescence. J Pediatr 2000: 136: 795-804.

13. Al-Qabandi W, Jenkinson HC, Buckels JA, et al. Orthotopic liver transplantation for unresectable hepatoblastoma: A single center's experience. J Pediatr Surg 1999: 34: 1261-1264.

14. Zsiros J, Maibach R, Shafford E, et al. Successful treatment of childhood high-risk hepatoblastoma with dose-intensive multiagent chemotherapy and surgery: Final results of the SIOPEL-3HR study. J Clin Oncol 2010: 28: 2584-2590.

15. Otte JB, Pritchard J, Aronson DC, et al. Liver transplantation for hepatoblastoma: Results from the International Society of Pediatric Oncology (SIOP) study SIOPEL-1 and review of the world experience. Pediatr Blood Cancer 2004: 42: 74-83.

16. Pimpalwar AP, Sharif K, Ramani P, et al. Strategy for hepatoblastoma management: Transplant versus nontransplant surgery. J Pediatr Surg 2002: 37: 240-245.

17. Avila L, Encinas J, Leal N, et al. Liver transplantation for malignant tumors in children. Cir Pediatr 2007: 20: 189-193.

18. Casas-Melley A, Malatack J, Consolini DJ, et al. Successful liver transplant for unresectable hepatoblastoma. J Pediatr Surg 2007: 42: 184-187.

19. Browne M, Sher D, Grant D, et al. Survival after liver transplantation for hepatoblastoma: A 2-center experience. J Pediatr Surg 2008: 43: 1973-1981.

20. Отте JB. Progress in the surgical treatment of malignant liver tumors in children. Cancer Treat Rev 2010: 36: 360-371

21. Malogolowkin MH, Katzenstein H, Krailo MD, et al. Intensified platinum therapy is an ineffective strategy for improving outcome in pediatric patients with advanced hepatoblastoma. J Clin Oncol 2006: 24: 2879-2884.

22. Warmann S, Fuchs J. Drug resistance in hepatoblastoma. Curr Pharm Biotechnol 2007: 8: 93-97.

23. Otte JB, Meyers R. PlutO first report. Pediatr Transplant 2010: 14: 830-835.

24. Darbari A, Sabin KM, Shapiro CN, Schwarz KB. Epidemiology of primary hepatic malignancies in U.S. children. Hepatology 2003: 38: 560-566.

25. Zimmermann A. Hepatoblastoma with cholangioblastic features ('cholangioblastic hepatoblastoma') and other liver tumors with bimodal differentiation in young patients. Med Pediatr Oncol 2002: 39: 487-491.

26. Zimmermann A. The emerging family of hepatoblastoma tumours: From ontogenesis to oncogenesis. Eur J Cancer 2005: 41: $1503-1514$

27. Katzenstein HM, Krailo MD, Malogolowkin MH, et al. Hepatocellular carcinoma in children and adolescents: Results from the Pediatric Oncology Group and the Children's Cancer Group intergroup study. J Clin Oncol 2002: 20: 2789-2797.

28. Czauderna P, Mackinlay G, Perilongo G, et al. Hepatocellular carcinoma in children: Results of the first prospective study of the International Society of Pediatric Oncology group. J Clin Oncol 2002: 20: 2798-2804.

29. Mazzaferro V, Regalia E, Doci R, et al. Liver transplantation for the treatment of small hepatocellular carcinomas in patients with cirrhosis. N Engl J Med 1996: 334: 693-699.

30. Prokurat A, Kluge P, Kosciesza A, Perek D, Kappeler A, Zimmermann A. Transitional liver cell tumors (TLCT) in older children and adolescents: A novel group of aggressive hepatic tumors expressing beta-catenin. Med Pediatr Oncol 2002: 39: $510-518$.

31. Beaunoyer M, Vanatta JM, Ogihara M, et al. Outcomes of transplantation in children with primary hepatic malignancy. Pediatr Transplant 2007: 11: 655-660.

32. Ismail H, Broniszczak D, Kalicinski $P$, et al. Liver transplantation in children with hepatocellular carcinoma. Do Milan criteria apply to pediatric patients? Pediatr Transplant 2009: 13: 682-692.

33. Gupta AA, Gerstle JT, NG V, et al. Critical review of controversial issues in the management of advanced pediatric liver tumors. Pediatr Blood Cancer 2011: 56: 1013-1018.

34. Mazariegos GV, Sindhi R, Thomson AW, Marcos A. Clinical tolerance following liver transplantation: Long term results and future prospects. Transpl Immunol 2007: 17: 114-119.

35. Koshiba T, Li Y, Takemura M, et al. Clinical, immunological, and pathological aspects of operational tolerance after pediatric living-donor liver transplantation. Transpl Immunol 2007: 17: 94-97.

36. Takatsuki M, Uemoto S, Inomata $\mathrm{Y}$, et al. Weaning of immunosuppression in living donor liver transplant recipients. Transplantation 2001: 72: 449-454.

37. Feng S, Ekong UD, Lobritto SJ, et al. Complete immunosuppression withdrawal and subsequent allograft function among pediatric recipients of parental living donor liver transplants. JAMA 2012: 307: 283-293.

38. AnsARI MJ, SAYEGH MH. Clinical transplantation tolerance: The promise and challenges. Kidney Int 2004: 65: 1560 1563.

39. Demetris AJ, Lunz JG III, Randhawa P, Wu T, Nalesnik M, THOMson AW. Monitoring of human liver and kidney allograft tolerance: A tissue/histopathology perspective. Transpl Int 2009: 22: 120-141.

40. Evans HM, Kelly DA, McKiernan PJ, Hubscher S. Progressive histological damage in liver allografts following pediatric liver transplantation. Hepatology 2006: 43: 1109-1117.

41. Scheenstra R, Peeters PM, Verkade HJ, Gouw AS. Graft fibrosis after pediatric liver transplantation: Ten years of follow-up. Hepatology 2009: 49: 880-886.

42. Abraham SC, Poterucha JJ, Rosen CB, Demetris AJ, KraSINSKAS AM. Histologic abnormalities are common in protocol liver allograft biopsies from patients with normal liver function tests. Am J Surg Pathol 2008: 32: 965-973.

43. Ekong UD, Melin-Aldana H, Seshadri R, et al. Graft histology characteristics in long-term survivors of pediatric liver transplantation. Liver Transpl 2008: 14: 1582-1587.

44. Mells G, Mann C, Hubscher S, Neuberger J. Late protocol liver biopsies in the liver allograft: A neglected investigation? Liver Transpl 2009: 15: 931-938.

45. NG VL, Fecteau A, Shepherd R, et al. Outcomes of 5-year survivors of pediatric liver transplantation: Report on 461 children from a north american multicenter registry. Pediatrics 2008: 122: e1128-e1135.

46. NG VL, Alonso EM, Bucuvalas JC, et al. Health status of children alive 10 years after pediatric liver transplantation performed in the US and Canada: Report of the studies of pediatric liver transplantation experience. J Pediatr 2012: 160: $820-826$ e 823.

47. Campbell $\mathrm{K}, \mathrm{Ng}_{\mathrm{V}} \mathrm{V}, \mathrm{Martin} \mathrm{S}$, et al. Glomerular filtration rate following pediatric liver transplantation-the SPLIT experience. Am J Transplant 2010: 10: 2673-2682.

48. Sundaram SS, Alonso EM, Zeitler P, Yin W, Anand R. Obesity after pediatric liver transplantation: Prevalence and risk factors. J Pediatr Gastroenterol Nutr 2012 Jun 28: PMID: 22744193.

49. Liem Ri, Anand R, Yin W, Alonso EM. Risk factors for chronic anemia in pediatric orthotopic liver transplantation: 


\section{Alonso et al.}

Analysis of data from the SPLIT registry. Pediatr Transplant 2012: 16: 137-143.

50. Mclin VA, Anand R, Daniels SR, Yin W, Alonso EM. Blood pressure elevation in long-term survivors of pediatric liver transplantation. Am J Transplant 2012: 12: 183-190.

51. Hathout E, Alonso E, Anand R, et al. Post-transplant diabetes mellitus in pediatric liver transplantation. Pediatr Transplant 2009: 13: 599-605.

52. McDiarmid SV, Anand R, Martz K, Millis MJ, Mazariegos G. A multivariate analysis of pre-, peri-, and post-transplant factors affecting outcome after pediatric liver transplantation. Ann Surg 2011: 254: 145-154.

53. La Greca AM, Bearman KJ. Adherence to pediatric treatment regimens. In: RoBERTS MC, ed. Handbook of Pediatric Psychology. New York: The Guilford Press, 2003: pp. 119140.

54. Shaw R. Treatment adherence in adolescents: Development and psychopathology. Clin Child Psychol Psychiatry 2001: 6: $137-150$.

55. Suris JC, Michaud PA, Viner R. The adolescent with a chronic condition. Part I: Developmental issues. Arch Dis Child 2004: 89: 938-942.

56. Dew MA, Dabbs AD, Myaskovsky L, et al. Meta-analysis of medical regimen adherence outcomes in pediatric solid organ transplantation. Transplantation 2009: 88: 736-746.

57. Dobbels F, Van Damme-Lombaert R, Vanhaecke J, De Geest S. Growing pains: Non-adherence with the immunosuppressive regimen in adolescent transplant recipients. Pediatr Transplant 2005: 9: 381-390.

58. Kahana SY, Frazier TW, Drotar D. Preliminary quantitative investigation of predictors of treatment non-adherence in pediatric transplantation: A brief report. Pediatr Transplant 2008: 12: 656-660.

59. Fredericks EM, Lopez MJ, Magee JC, Shieck V, OpipariArrigan L. Psychological functioning, nonadherence and health outcomes after pediatric liver transplantation. Am J Transplant 2007: 7: 1974-1983.

60. Fredericks eM, Magee JC, Opipari-Arrigan L, Shieck V, Well A, Lopez MJ. Adherence and health-related quality of life in adolescent liver transplant recipients. Pediatr Transplant 2008: 12: 289-299.

61. Griffin KJ, Elkin TD. Non-adherence in pediatric transplantation: A review of the existing literature. Pediatr Transplant 2001: 5: 246-249.

62. Pinsky BW, Takemoto SK, Lentine KL, Burroughs Te, Schnitzler MA, Salvalaggio PR. Transplant outcomes and economic costs associated with patient noncompliance to immunosuppression. Am J Transplant 2009: 9: 2597-2606.
63. WATSON AR. Non-compliance and transfer from paediatric to adult transplant unit. Pediatr Nephrol 2000: 14: 469-472.

64. Annunziato RA, Emre S, Shneider B, Barton C, Dugan CA, Shemesh E. Adherence and medical outcomes in pediatric liver transplant recipients who transition to adult services. Pediatr Transplant 2007: 11: 608-614.

65. Fine RN, Becker Y, De Geest $\mathrm{S}$, et al. Nonadherence consensus conference summary report. Am J Transplant 2009: 9: 3541.

66. Bucuvalas JC, Ryckman FC, Arya G, et al. A novel approach to managing variation: Outpatient therapeutic monitoring of calcineurin inhibitor blood levels in liver transplant recipients. J Pediatr 2005: 146: 744-750.

67. Shemesh E, Shneider BL, Savitzky JK, et al. Medication adherence in pediatric and adolescent liver transplant recipients. Pediatrics 2004: 113: 825-832.

68. Stuber ML, Shemesh E, Seacord D, Washington J III, HelLEMANN G, McDiarmid S. Evaluating non-adherence to immunosuppressant medications in pediatric liver transplant recipients. Pediatr Transplant 2008: 12: 284-288.

69. Blum RW, Garell D, Hodgman CH, et al. Transition from child-centered to adult health-care systems for adolescents with chronic conditions. A position paper of the Society for Adolescent Medicine. J Adolesc Health 1993: 14: 570-576.

70. Kennedy A, Sawyer S. Transition from pediatric to adult services: Are we getting it right? Curr Opin Pediatr 2008: 20: 403409.

71. Sawyer SM, Blair S, Bowes G. Chronic illness in adolescents: Transfer or transition to adult services? J Paediatr Child Health 1997: 33: 88-90.

72. Kieckhefer GM, Trahms CM. Supporting development of children with chronic conditions: From compliance toward shared management. Pediatr Nurs 2000: 26: 354-363.

73. Watson AR. Problems and pitfalls of transition from paediatric to adult renal care. Pediatr Nephrol 2005: 20: 113-117.

74. Fredericks EM, Dore-Stites D, Well A, et al. Assessment of transition readiness skills and adherence in pediatric liver transplant recipients. Pediatr Transplant 2010: 14: 944-953.

75. McDonagh JE, HaCKetT J. Interrelationship of self-management and transitional care needs of adolescents with arthritis: Comment on the article by Stinson et al. Arthritis Rheum 2008: 59: 1199-1200; author reply 1200-1191.

76. VINER R. Transition from paediatric to adult care. Bridging the gaps or passing the buck? Arch Dis Child 1999: 81: 271-275.

77. McDonagh JE, Kelly DA. Trans-plan-sition! Transplantation and transition. Pediatr Transplant 2007: 11: 578-581. 